\title{
Piezo-electrically Actuated Micro Corner Cube Retroreflector (CCR) for Free-space Optical Communication Applications
}

\author{
Duk-Hyun Lee ${ }^{\dagger}$ and Jae Y. Park*
}

\begin{abstract}
In this paper, an extremely low voltage operated micro corner cube retroreflector (CCR) was fabricated for free-space optical communication applications by using bulk silicon micromachining technologies. The CCR was comprised of an orthogonal vertical mirror and a horizontal actuated mirror. For low voltage operation, the horizontal actuated mirror was designed with two PZT cantilever actuators, torsional bars, hinges, and a mirror plate with a size of $400 \mu \mathrm{m} \times 400 \mu \mathrm{m}$. In particular, the torsional bars and hinges were carefully simulated and designed to secure the flatness of the mirror plate by using a finite element method (FEM) simulator. The measured tilting angle was approximately $2^{\circ}$ at the applied voltage of $5 \mathrm{~V}$. An orthogonal vertical mirror with an extremely smooth surface texture was fabricated using $\mathrm{KOH}$ wet etching and a double-SOI (silicon-on-insulator) wafer with a (110) silicon wafer. The fabricated orthogonal vertical mirror was comprised of four pairs of two mutually orthogonal flat mirrors with $400 \mu \mathrm{m}$ (length) $\times 400 \mu \mathrm{m}$ (height) $\times 30 \mu \mathrm{m}$ (thickness). The cross angles and surface roughness of the orthogonal vertical mirror were orthogonal, almost $90^{\circ}$ and $3.523 \mathrm{~nm}$ rms, respectively. The proposed CCR was completed by combining the orthogonal vertical and horizontal actuated mirrors. Data transmission and modulation at a frequency of $10 \mathrm{~Hz}$ was successfully demonstrated using the fabricated CCR at a distance of approximately $50 \mathrm{~cm}$.
\end{abstract}

Keywords: MEMS, Orthogonal mirror, Corner cube retroreflector (CCR), Vertical silicon mirror, Piezoelectric actuation, Cantilever, Optical communication

\section{Introduction}

Although recent technological advances in micro electro-mechanical systems (MEMS) promoted wireless sensor network devices, these devices needed irreplaceable power sources to operate the sensor nodes for practical applications [1]-[4]. To overcome these obstacles, passive reflective systems have been developed for wireless sensor nodes [5]-[10]. Although these sensor nodes did not have light sources, they transmitted data only by reflecting external light using reflectors. Therefore, they are very attractive for transceivers to improve the power efficiency of the sensor nodes. Additionally, the high directionality and narrow beam of optical transceivers provide greater promise to secure the signal from wiretap, thus, they are suitable for military and security applications. These reflectors are called micro comer cube retroreflectors (CCR), which was first proposed in the smart dust project [4].

Most of the CCRs, previously reported, were operated with a magnetic and electrostatic actuation mechanism to actuate the horizontal mirrors. When an external magnetic field was applied to magnetic elements beside CCR, the actuated mirror attached to the magnetic elements was rotated [6]. There were some difficulties such as the fabrication and miniaturization of CCR due to the extra magnetic

\footnotetext{
$\dagger \quad$ Corresponding Author: Dept. of Electronic Engineering, Kwangwoon University, Korea. (dukyuny@gmail.com)

* Dept. of Electronic Engineering, Kwangwoon University, Korea. (jaepark@kw.ac.kr)

Received: February 16, 2010; Accepted: April 9, 2010
}

elements. CCRs with electro-statically actuated mirrors need high driving voltages ranging from $20 \mathrm{~V}$ to $50 \mathrm{~V}$ [7][9]. Therefore, both of these actuation mechanisms were not appropriate for the wireless sensor node systems due to their high operating voltages and bulky size.

In the early stages, CCRs used large passive mirrors fabricated by surface micromachining technology to form an orthogonal vertical mirror [6]-[7]. These suffered from mirror curvature due to asymmetric film stresses, or require manual assembly which made it easy to misalign two mutual mirrors. The orthogonal vertical mirror, recently published, was fabricated using $\mathrm{KOH}+$ IPA after DRIE etching [9]. This fabrication process could prevent misalignment, although the fabrication process might be highly expensive and complicated. In addition, its surface texture was not good enough.

In this paper, new design and fabrication methods were proposed for developing cost effective CCR with extremely low voltage operation and negligible power consumption. PZT cantilever actuators were utilized to fabricate a horizontal actuated mirror for low voltage operation. Also, an orthogonal vertical MEMS mirror with extremely smooth surface roughness was easily fabricated using $\mathrm{KOH}$ wet etching and a double-SOI wafer. The CCR was completed by aligning and combining the orthogonal vertical and horizontal actuated mirrors. 


\section{Design and Fabrication}

A CCR is an optical device consisting of a horizontal actuated mirror and an orthogonal vertical mirror and it forms a concave corner with mutually orthogonal mirrors as shown Fig 1. In the on-state, each mirror is completely orthogonal to the other two mirrors, and the entered light is then reflected in parallel to the source as shown Fig. 1 (a). When the horizontal actuated mirror is tilted to the other vertical mirrors as shown Fig. 1 (b), the returned light is reflected in a different direction to the incident light. As these are simple operations, the CCR digitally modulates the light for communication by either reflecting an external laser back towards the source or by scattering the laser away from the source.

The horizontal actuated mirror was optimally designed and simulated using an FEM simulator (CoventorWare) before fabrication. The horizontal actuated mirror consisted of a mirror plate of $400 \mu \mathrm{m} \times 400 \mu \mathrm{m}$, PZT cantilever actuators, hinges, and torsional bars. The PZT cantilever actuators were connected to the edge of the mirror plate using the hinges. The mirror plate was suspended by torsional bars from substrate. The structural geometry and dimension of the hinges and torsional bars were also optimized to secure the flatness of the mirror plate on the actuation mode. The designed horizontal actuated mirror was operated by applying dc voltages at the top and bottom elec-

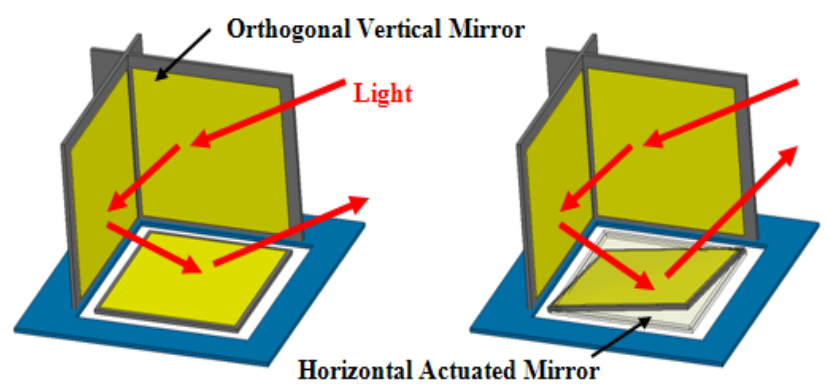

(a)

(b)

Fig. 1. Schematic drawings of the proposed CCR operation principle : (a) on state and (b) off state.

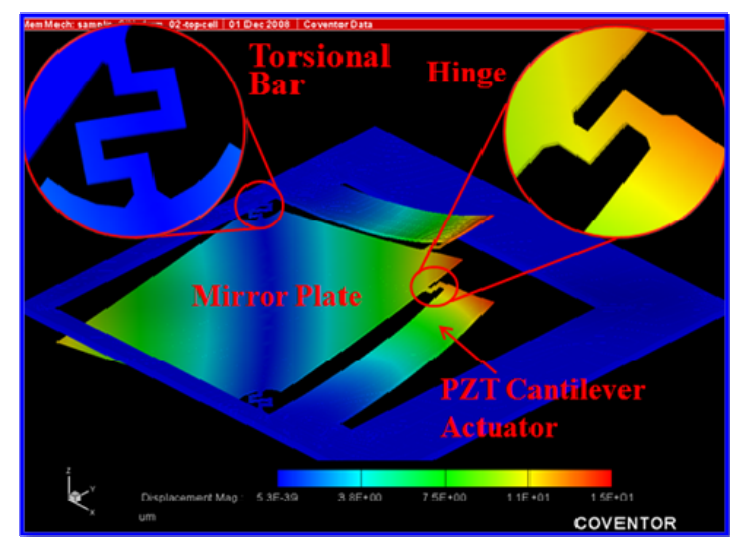

Fig. 2. Simulation result of the horizontal actuated mirror at the applied voltage of $5 \mathrm{~V}$. trode of the PZT cantilever actuators. Fig. 2 shows the actuation mode of the horizontal actuated mirror with five voltages applied. In simulation, the horizontal actuated mirror was rotated along the diagonal axis with two torsional bars of the mirror plate edges.

The fabrication processes of the proposed CCR were divided into three parts. The first part was to fabricate the horizontal actuated mirror with PZT cantilever actuators, hinges and torsional bars. The second part was to fabricate the orthogonal vertical mirror by using a double SOI wafer and $\mathrm{KOH}$ wet etching. In the last part, the horizontal actuated and the orthogonal vertical mirrors were aligned and assembled using SU-8 holders.

Fig. 3 (a) shows the fabrication process for a horizontal actuated mirror. $1 \mu \mathrm{m}$ thick low stress silicon nitride layer was coated on a (100) silicon substrate of $500 \mu \mathrm{m}$ thickness and then Ti/Pt bottom electrode, PZT, and Pt top electrode were sequentially deposited on the low stress silicon nitride. The mirror plate, hinges, and torsional bars were formed

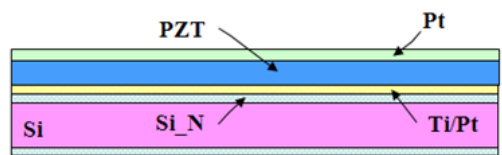

(1)
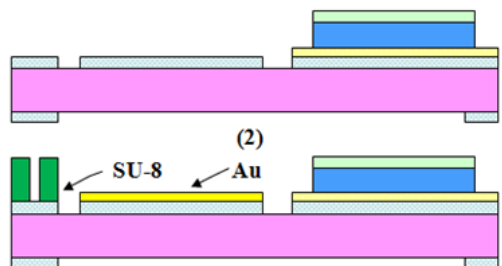

(3)

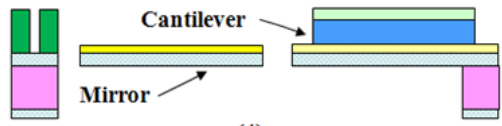

(4)

(a)

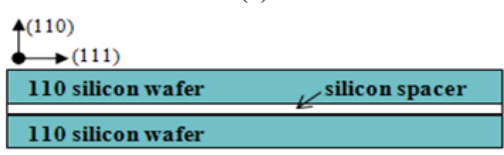

(1)

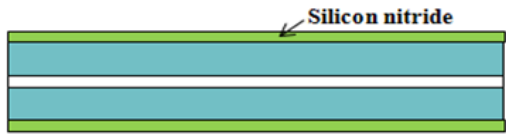

(2)

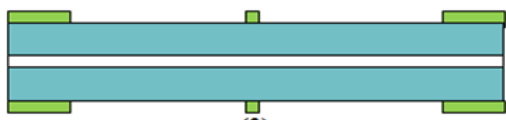

(3)

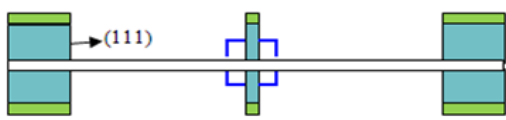

(4)

(b)

Fig. 3. Fabrication processes of (a) the horizontal actuated mirror and (b) the orthogonal vertical silicon mirror. 
from $1 \mu \mathrm{m}$ thick low stress silicon nitride layer on buried oxide of $5000 \AA$. The PZT cantilever actuators consisted of Ti/Pt bottom electrode, PZT, and Pt top electrode, which were sequentially deposited on the low stress silicon nitride. Gold and SU-8 were deposited to improve the reflectivity of the mirror plate and to align the orthogonal vertical mirror. Using the buried $\mathrm{SiO} 2$ layer under the silicon nitride as an etch stop layer, silicon substrate was selectively etched using the backside DRIE process to release the horizontal actuated mirror. The fabricated horizontal actuated mirror is shown in Fig. 4 (a).

Fig. 3 (b) shows the fabrication procedure for the orthogonal vertical silicon mirror. The starting material was a double-SOI (silicon-on-insulator) wafer with top and bottom (110) silicon wafers $400 \mu \mathrm{m}$ thick and a $30 \mu \mathrm{m}$ thick center silicon spacer using a (100) silicon wafer. A $1 \mu \mathrm{m}$ thick insulating $\mathrm{SiO}_{2}$ layer was used between the silicon wafers. On the double-SOI wafer, LPCVD silicon nitride film of $2000 \AA$ was coated to make layer in the $\mathrm{KOH}$ wet etching. Then, top and bottom silicon nitride layers were sequentially patterned using the same mask with parallel lines to the (111) direction. Next, a double-SOI wafer was etched down to $400 \mu \mathrm{m}$ as far as $\mathrm{SiO}_{2}$ and used for the etch stop layer using $\mathrm{KOH}$ solution. After that, a pair of vertical comb structures was symmetrically formed with highaspect-ratio. To minimize the surface roughness of the formed silicon mirrors, $\mathrm{KOH}$ etchant was optimized as $40 \%$ concentration and $70^{\circ} \mathrm{C}$ of processing temperature. After $\mathrm{KOH}$ wet etching, the etched double-SOI wafer was sawed in a direction perpendicular to the formed structures.

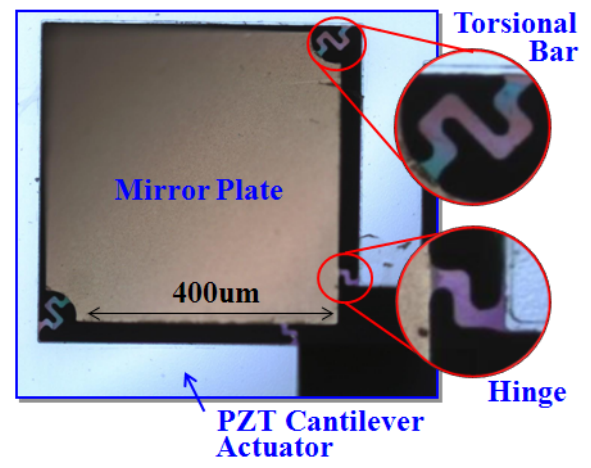

(a)

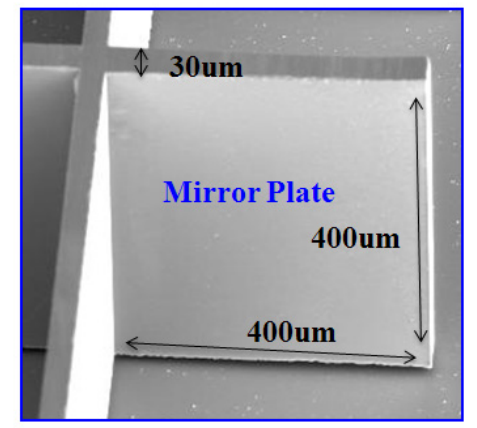

(b)

Fig. 4. Photomicrographs of (a) the horizontal actuated and (b) orthogonal vertical mirrors.
Fig. 4 (a) shows the SEM picture of the fabricated orthogonal vertical mirror with $400 \mu \mathrm{m}$ (length) $\times 400 \mu \mathrm{m}$ (height) $\times 30 \mu \mathrm{m}$ (thickness).

Finally, the CCR was completed by aligning and inserting the orthogonal vertical mirror between the SU-8 holders. The SU-8 micro-holders are patterned on top of the silicon substrate from which the horizontal actuated mirror was fabricated. Fig. 5 shows the SEM picture of the fully assembled CCR device.

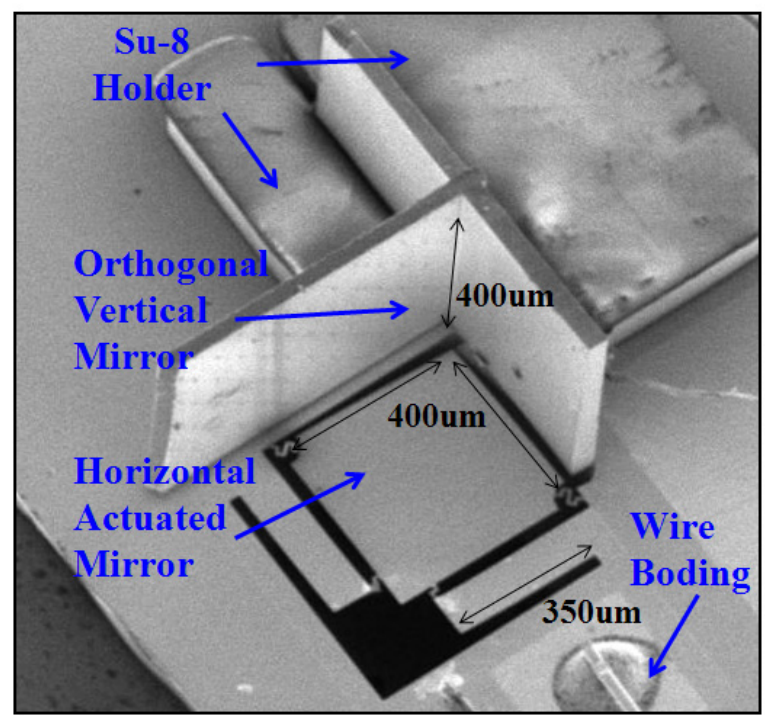

Fig. 5. SEM picture of the fabricated CCR.

\section{Measurements and Results}

The tilting angle was measured using an optical test system as shown in Fig. 6 (a). The laser beam was focused on the mirror plate of the horizontal actuated mirror. The laser beam spot reflected from the mirror plate was incident at the $1^{\text {st }}$ point. When the horizontal actuated mirror was tilted up by applying dc voltages, the reflected laser beam spot moved to the $2^{\text {nd }}$ point. The moved laser beam spot was relocated by adjusting the rotation stage to the $1^{\text {st }}$ point. The moved angle at the rotation stage was the tiling angle of the horizontal actuated mirror. Fig. 6 (b) shows the measured tilting angles with respect to the applied voltages. The tilting angle of the horizontal actuated mirror was roughly $2^{\circ}$ at $5 \mathrm{~V}$. The tilting angle over $1.4^{\circ}$ was sufficient through numerical calculation using a trigonometric function, when the distance was $10 \mathrm{~m}$ from the CCR to the sensor detector. As shown in Fig. 6 (b), the measured tilting angles were well agreed with the simulated ones at the low applied voltages. However, there were some discrepancies between the measured and simulated angles as the applied voltages increased. Since the tilting angle was physically limited, it might be reduced as much as the initial bending of the PZT cantilever actuators caused by the residual stress.

Fig. 7 shows the top view of the fabricated orthogonal 


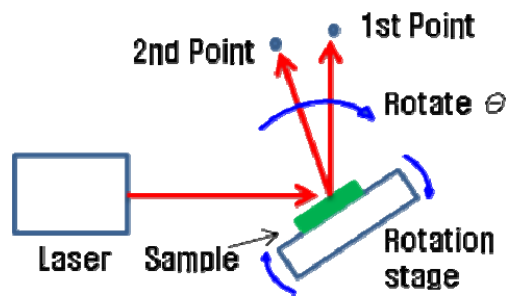

(a)

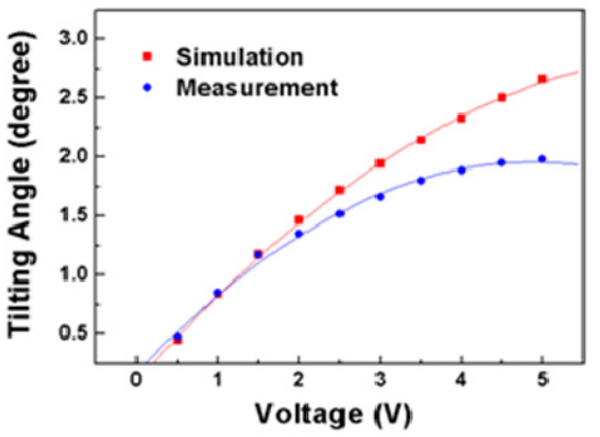

(b)

Fig. 6. (a) Schematic drawing of optical set up for testing the tilting angle and (b) the simulated and measured results of the horizontal actuated mirror at applied dc voltages.

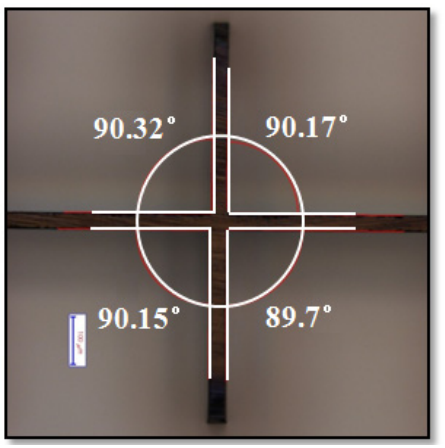

(a)

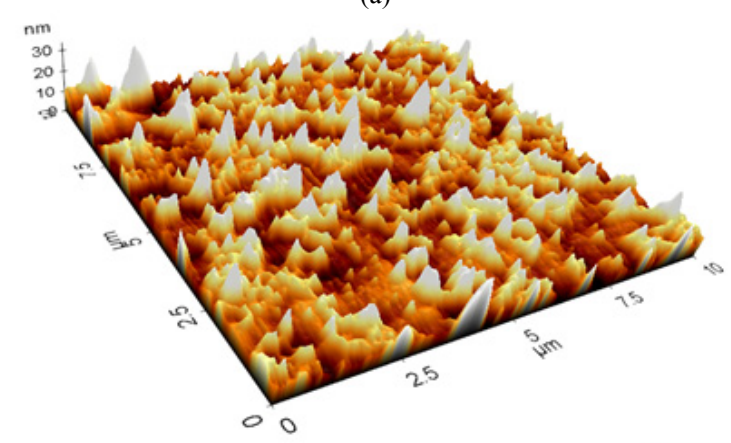

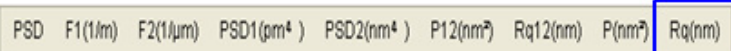

\begin{tabular}{|c|c|c|c|c|c|c|}
\hline \pm 20 & 0.000 & 12.800 & OEO & $6.165 \mathrm{E} 0$ & 12.410 & 3.523 12.41d \\
\hline
\end{tabular}

(b)

Fig. 7. (a) Top view of the fabricated orthogonal vertical silicon mirror and its cross angles and (b) AFM measurement of the mirror surface.

vertical silicon mirror captured using an optical microscope.
The measured maximum and minimum cross angles were $90.32^{\circ}$ and $89.7^{\circ}$, respectively. The variation of cross angle was bounded within $0.4^{\circ}$ and its average angle was $90.09^{\circ}$. The surface roughness of the mirror plate was measured by using AFM and it exhibited $3.523 \mathrm{~nm}$ rms (root-meansquare) as shown in Fig. 5. At the surface roughness of $3.523 \mathrm{~nm} \mathrm{rms}$, the amount of scattering loss was calculated for different incident angles and wavelengths. The calculated results were summarized in Table 1 . The maximum scattering light was below $0.5 \%$, when a He-Ne laser $(\lambda=$ $632.8 \mathrm{~nm}$ ) was applied into the perpendicular direction on the mirror plate $\left(\theta=0^{\circ}\right)$ [10].

Optical performance of the fabricated CCR was measured using the laser reflection method. A silicon photodiode was used to detect a HeNe laser beam reflected from the CCR. The distance from the CCR to the photodetector was $50 \mathrm{~cm}$. When the CCR was driven from a signal generator using a $5 \mathrm{~V}$ square wave at a frequency of $10 \mathrm{~Hz}$, the optical signal generated was then detected by the photodiode and passed to an oscilloscope. Fig. 8 shows the detected signal obtained when the CCR modulated the reflected light.

Table 1. Calculated amount of scattering loss at surface roughness of $3.523 \mathrm{~nm} \mathrm{rms}$

\begin{tabular}{c|c|c|c|c}
\hline \multicolumn{2}{c|}{} & \multicolumn{3}{|c}{ Wavelength } \\
\cline { 3 - 5 } \multicolumn{2}{c}{} & $632.8 \mathrm{~nm}$ & $1310 \mathrm{~nm}$ & $1550 \mathrm{~nm}$ \\
\hline \multirow{2}{*}{$\begin{array}{c}\text { Incident } \\
\text { Angle }\end{array}$} & $\theta=0^{\circ}$ & $0.49 \%$ & $0.11 \%$ & $0.08 \%$ \\
\cline { 2 - 5 } & $\theta=45^{\circ}$ & $0.24 \%$ & $0.06 \%$ & $0.04 \%$ \\
\hline
\end{tabular}

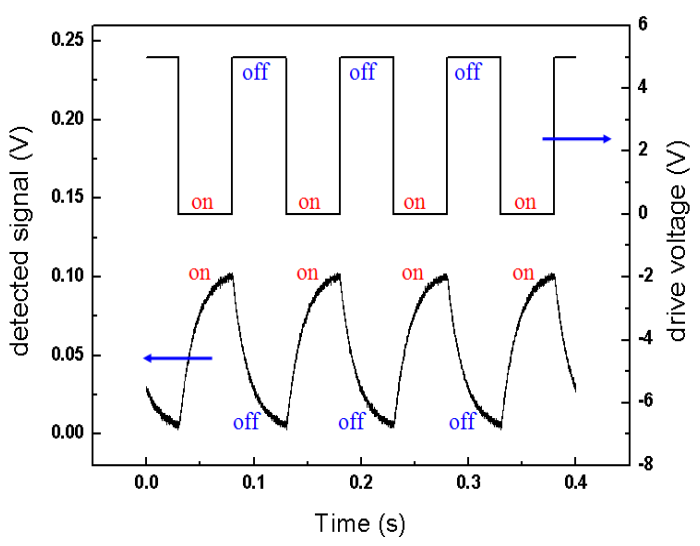

Fig. 8. Step response of the fabricated CCR by applying 10 $\mathrm{Hz}$ square-wave voltages of $5 \mathrm{~V}$.

\section{Conclusion}

A low voltage operated MEMS Corner Cube Retroreflector (CCR) was newly fabricated for free-space optical communication and micro-optical system applications. The fabricated CCR consisted of orthogonally vertical and horizontal actuated mirrors with a size of $400 \mu \mathrm{m} \times 400 \mu \mathrm{m}$. The horizontal actuated mirror was fabricated using piezoelectric cantilever actuators for low voltage operation and negligible power consumption. The orthogonally vertical 
silicon mirror was newly developed using a double-SOI wafer with (110) silicon wafer in order to overcome some restrictions of design in the $\mathrm{KOH}$ wet etching. The proposed CCR was fabricated by aligning and inserting the orthogonal vertical silicon mirror between SU-8 holders formed onto the silicon substrate which the horizontal actuated mirror was fabricated. The measured tilting angle of the horizontal actuated mirror was approximately $2^{\circ}$ at $5 \mathrm{~V}$, which was good enough to satisfy the optical communication at a distance of $10 \mathrm{~m}$ between the CCR and a sensor detector with a size of $10 \mathrm{~cm}$. The fabricated orthogonal vertical mirror exhibited extremely smooth surface roughness and $90^{\circ}$ cross angles. Data transmission and modulation at a frequency of $10 \mathrm{~Hz}$ was successfully demonstrated using the CCR at a distance of approximately $50 \mathrm{~cm}$. However, the bottom actuated mirror was not perfectly flat in the measured result because of the residual stress gradients in the stacked film layers. In the near future, these undesirable factors will be addressed through the optimization of the material selection and the process conditions.

\section{Acknowledgements}

This research was financially supported by a grant to MEMS Research Center for National Defense funded by Defense Acquisition Program Administration. The authors wish to thank MiNDaP group members for their technical supports and discussions.

\section{References}

[1] D. Steere, A. Baptista, D. McNamee, C. Pu and J. Walpole, "Research challenges in environmental observation and forecasting systems," in Proc. ACM/IEEE MOBICOM, pp. 292-299, Aug. 2000.

[2] L. Schwiebert, S. K. S. Gupta and J. Weinmann, "Research challenges in wireless networks of biomedical sensors," in Proc. ACM/IEEE MOBICOM, pp. 151165, 2001.

[3] S. D. Feller, E. Cull, D. P. Kowalski, K. Farlow, J. Burchett, J. Adleman, C. Lin and D. J. Brady, "Tracking and imaging humans on heterogeneous infrared sensor array for tactical applications," SPIE Aerosense, Apr. 2002.

[4] J. Kahn, R. H. Katz and K. Pister, "Emerging Challenges: Mobile Networking for 'Smart Dust'," $J$. Communications and Networks, Vol. 2, No. 3, pp. 188-196, Sep., 2000.

[5] S. Teramoto and T. Ohtsuki, "OpticalWireless Sensor Network System Using Corner Cube Retroreflectors," J. Wireless Communications and Networking, Vol. 1, pp. 39-44, 2005.

[6] D. J. Vasquez and Jack W. Judy, "OpticallyInterrogated Zero-Power MEMS Magnetometer," $J$. Microelecmech. Syst., Vol. 16, No.2, Apr. 2007.

[7] L. Zhou, J. M. Kahn and K. S. J. Pister, "Corner-cube retroreflectors based on structure-assisted assembly for free-space optical communication," J. Microelectromech. Syst., Vol. 12, No. 3, pp. 233-242, June 2003.

[8] Y. K. Hong and R. R. A. Syms, "Dynamic Response Modeling of MEMS Micromirror Corner Cube Reflectors With Angular Vertical Combdrives," $J$. Lightwave Technol., Vol. 25, pp. 472-480, 2007.

[9] R. Agarwal, S. Samson, S. Kedia and S. Bhansali, "Fabrication of Integrated Vertical Mirror Surfaces and Transparent Window for Packaging MEMS Devices," J. Microelectromech. Syst., Vol. 16, No. 1, Feb. 2007.

[10] C. Marxer, C. Thio, M. Gretillat, N. F. de Rooji, R. Battig, R. Anthamatten, B. Valk and P. Vogel, "Vertical mirrors fabricated by deep reactive ion etching for fiber-optic switching applications," J. Microelectromech. Syst., Vol. 6, No. 7, pp. 277-285, July 1997.

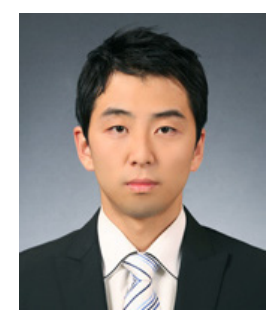

Duk-Hyun Lee received his B.S. degree from the Department of Wireless Communications Engineering from Kwangwoon University, Seoul, Korea, in 2008. He received his M.S. degree from the Department of Electronic Engineering at Kwangwoon University, Seoul, Korea, in 2010. His research interests are microelectronic devices, optical MEMS, and packaging.

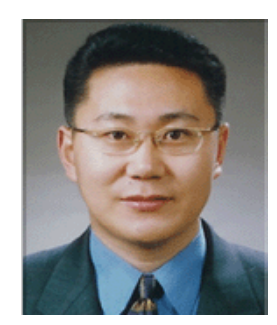

Jae-Yeong Park received his B.S. degree from the Department of Telecommunication Engineering from Korea Aerospace University, Seoul, Korea, in 1992. He received his M.S.E.E. and Ph.D. degrees from the School of Electrical and Computer Engineering from Georgia Institute of Technology, Atlanta, GA, USA, in 1995 and 1997, respectively. After graduation, he worked at the Packaging Research Center of Georgia Institute of Technology as a research engineer for two years. He has also worked at Microsystem Group in LG Electronics Institute of Technology as a Team leader of RF MEMS research for six years. In September 2004, he joined the faculty of the department of Electronics Engineering in Kwangwoon University in Seoul, Korea. He has published more than 150 journal articles and conference proceedings and filed more than 105 patents. His current research interests include vibration based MEMS energy harvesting devices, microactuators for optical and RF applications, micro-packaging (wafer level packaging and micro-solder transfering), nonenzymatic electrochemical bio and environmental sensors, and PCB embedding RF components and modules. 